
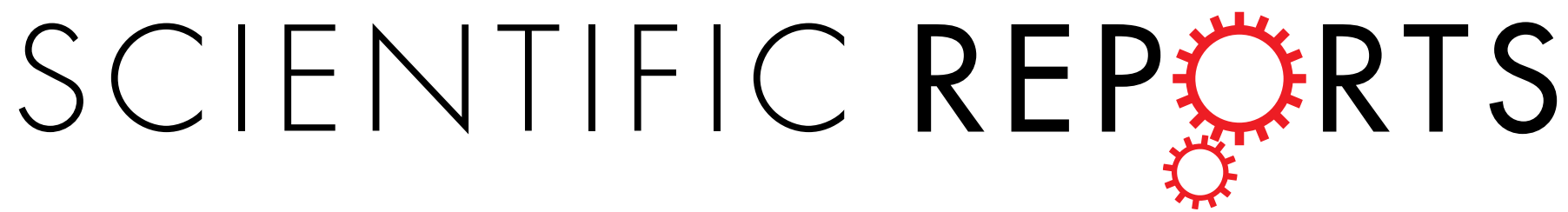

OPEN

Received: 17 October 2016

Accepted: 17 March 2017

Published: 19 April 2017

\section{Detection of a new pyrethroid} resistance mutation (V410L) in the sodium channel of Aedes aegypti: a potential challenge for mosquito control

\author{
Khalid Haddi', ${ }^{1}$, Hudson V. V. Tomé ${ }^{1,3}$, Yuzhe Du ${ }^{4}$, Wilson R. Valbon ${ }^{1}$, Yoshiko Nomura ${ }^{4}$, \\ Gustavo F. Martins ${ }^{5}$, Ke Dong ${ }^{4}$ \& Eugênio E. Oliveira ${ }^{1}$
}

The yellow fever mosquito, Aedes aegypti, particularly in Neotropical regions, is the principal vector of dengue, yellow fever, Zika and Chikungunya viruses. Pyrethroids remain one of the most used insecticides to control Aedes mosquitoes, despite the development of pyrethroid resistance in many mosquito populations worldwide. Here, we report a Brazilian strain of $A$. aegypti with high levels (approximately 100-60,000 fold) of resistance to both type I and type II pyrethroids. We detected two mutations (V410L and F1534C) in the sodium channel from this resistant strain. This study is the first report of the V410L mutation in mosquitoes. Alone or in combination with the $\mathrm{F} 1534 \mathrm{C}$ mutation, the V410L mutation drastically reduced the sensitivity of mosquito sodium channels expressed in Xenopus oocytes to both type I and type II pyrethroids. The V410 L mutation presents a serious challenge for the control of $A$. aegypti and will compromise the use of pyrethroids for the control of $A$. aegypti in Brazil; therefore, early monitoring of the frequency of the $\mathrm{V} 410 \mathrm{~L}$ mutation will be a key resistance management strategy to preserve the effectiveness of pyrethroid insecticides.

With vaccines unavailable for most of the arboviruses transmitted by mosquitoes, vector control has long been a key strategy to manage mosquito-associated diseases. The essential focus of this strategy is the elimination of breeding sites and the application of insecticides against both adults and larvae ${ }^{1-5}$. Our current mosquito control strategies have been the target of harsh condemnation ${ }^{2}$, mostly based on the spectacular increase in the global prevalence and incidence of dengue (DENV) and more recently, chikungunya (CHIKV) and Zika (ZIKV) arboviruses. For example, as of June 2016, 61 countries and territories report continuing Zika mosquito-borne transmission, with 47 experiencing a first outbreak of Zika virus ${ }^{4}$. In Brazil, more than 1600 cases of microcephaly and/or central nervous system malformation have been confirmed that are potentially associated with Zika virus infection ${ }^{4}$.

Aedes aegypti is the primary vector for Zika and dengue worldwide and also has high vectorial capacity for chikungunya and yellow fever arboviruses ${ }^{2,4,5}$. The prevention or reduction of transmission of these arboviruses is mostly dependent on the control of mosquitoes and limiting their contacts with humans. Among the various compounds (e.g., organophosphates and neonicotinoids for control of larvae and adults and insect growth regulators (IGR) and Bacillus-based compounds for control of larvae) recommended by the World Health Organization (WHO) in the effort to control mosquitoes, pyrethroid insecticides are one of most important because of their efficacy and safety ${ }^{1-5}$.

${ }^{1}$ Departamento de Entomologia, Universidade Federal de Viçosa, Viçosa, MG 36570-900, Brasil. ${ }^{2}$ Science Without Border Associate Researcher, Programa de Pós-Graduação em Entomologia, Universidade Federal de Viçosa, Viçosa, MG 36570-000, Brasil. 'EAG Laboratories, 13709 Progress Blvd. \#24 Suite S163, Alachua, FL, 32615 USA. ${ }^{4}$ Department of Entomology, Genetics and Neuroscience Programs, Michigan State University, East Lansing, MI, USA. ${ }^{5}$ Departamento de Biologia Geral, Universidade Federal de Viçosa, Viçosa, M G 36570-900, Brasil. Correspondence and requests for materials should be addressed to K.H. (email: khalid.haddi@ufv.br) or E.E.O. (email: eugenio@ufv.br) 


\begin{tabular}{|c|c|c|c|c|c|c|c|c|}
\hline $\begin{array}{l}\text { Insect } \\
\text { phase }\end{array}$ & Insecticide & Population & $n$ & Slope \pm S.E & $\begin{array}{c}\mathrm{LC}_{50}(95 \% \mathrm{CI}) \text { larvae } \\
(\mu \mathrm{g} \text { a.i. } / \mathrm{mL}) \text { adults }\left(\mu \mathrm{g} \text { a.i. } / \mathrm{cm}^{2}\right)\end{array}$ & $\chi^{2}$ & $P$-values ${ }^{a}$ & $\begin{array}{l}R^{b} \mathrm{LC}_{50} \\
(95 \% \mathrm{CI})^{c}\end{array}$ \\
\hline \multirow{4}{*}{$\begin{array}{l}\text { Larvae } \\
\text { (L4) }\end{array}$} & \multirow{2}{*}{$\begin{array}{l}\text { Permethrin } \\
\quad \text { (type I } \\
\text { pyrethroid) }\end{array}$} & susceptible & 600 & $16.2 \pm 5.05$ & $\begin{array}{c}1.1 \times 10^{-3}\left(9.8 \times 10^{-4}\right. \\
\left.-1.5 \times 10^{-3}\right)\end{array}$ & 7.07 & 0.13 & - \\
\hline & & resistant & 500 & $1.7 \pm 0.15$ & $64.7(46.31-89.54)$ & 0.30 & 0.95 & $\begin{array}{c}59830.1 \\
(38150.1-93830.4)\end{array}$ \\
\hline & \multirow{2}{*}{$\begin{array}{l}\text { Deltamethrin } \\
\text { (type II } \\
\text { pyrethroid) }\end{array}$} & susceptible & 420 & $4.4 \pm 0.56$ & $\begin{array}{c}2.7 \times 10^{-3}\left(2.2 \times 10^{-3}\right. \\
\left.-3.3 \times 10^{-3}\right)\end{array}$ & 1.92 & 0.59 & - \\
\hline & & resistant & 420 & $0.7 \pm 0.13$ & $4.4(2.94-6.18)$ & 6.23 & 0.18 & $\begin{array}{c}1568.0 \\
(772.6-3186.2) \\
\end{array}$ \\
\hline \multirow{4}{*}{ Adults } & \multirow{2}{*}{$\begin{array}{l}\text { Permethrin } \\
\quad \text { (type I } \\
\text { pyrethroid) }\end{array}$} & susceptible & 591 & $2.6 \pm 0.28$ & $\begin{array}{c}4.3 \times 10^{-3}\left(4.0 \times 10^{-3}\right. \\
\left.-4.5 \times 10^{-3}\right)\end{array}$ & 5.04 & 0.17 & - \\
\hline & & resistant & 809 & $4.0 \pm 0.27$ & $0.35(0.32-0.38)$ & 6.51 & 0.55 & $102.2(73.7-141.7)$ \\
\hline & \multirow{2}{*}{$\begin{array}{l}\text { Deltamethrin } \\
\text { (type II } \\
\text { pyrethroid) }\end{array}$} & susceptible & 671 & $5.3 \pm 0.39$ & $\begin{array}{c}7.6 \times 10^{-6}\left(6.6 \times 10^{-6}\right. \\
\left.-8.8 \times 10^{-6}\right)\end{array}$ & 5.67 & 0.23 & - \\
\hline & & resistant & 562 & $1.8 \pm 0.13$ & $\begin{array}{c}9.8 \times 10^{-4}\left(8.2 \times 10^{-4}\right. \\
\left.-1.2 \times 10^{-3}\right)\end{array}$ & 2.62 & 0.60 & $128.9(88.4-188.3)$ \\
\hline
\end{tabular}

Table 1. Relative toxicity of type I and type II pyrethroids to larvae and adults of two Brazilian strains of Aedes aegypti. ${ }^{\text {PProbability values. }}{ }^{b}$ Resistance ratio $\left(\mathrm{LC}_{50}\right.$ of resistant population/LC $\mathrm{L}_{50}$ of susceptible population). ${ }^{c}$ When the $95 \%$ CI of RR included 1.0, the RRs were not significantly different (Robertson and Preisler, 1992).

Pyrethroids exert their toxic effects by disrupting the functionalities of the voltage-gated sodium channels in the insect nervous system ${ }^{6-9}$. However, the development of pyrethroid resistance is the primary threat to the sustained use of pyrethroids for mosquito control ${ }^{8-12}$. A well-known mechanism of pyrethroid resistance, knockdown resistance ( $\mathrm{kdr}$ ), is caused by naturally occurring mutations in the voltage-gated sodium channel, which occur in a wide range of medically and agriculturally important arthropods, including disease vectors ${ }^{7,10,13-15}$. Several kdr mutations are recorded in the sodium channels of $A$. aegypti populations ${ }^{10,16-18}$, but only some of these mutations reduce the sodium channel sensitivity to pyrethroids $s^{7,11,19,20}$.

Thus, understanding the molecular bases of pyrethroid resistance in A. aegypti is crucial for early detection and monitoring of the resistance phenomenon, and to the development of appropriate strategies to delay the occurrence and further spread of resistance. Here, in two Brazilian strains of A. aegypti, we evaluated the susceptibility and then demonstrated the contributions of metabolic and site-target alteration mechanisms in limiting the actions of pyrethroid insecticides. For the first time in mosquitoes, we describe a valine to leucine substitution (V410L) in the transmembrane segment 6 of domain I that contributed to high levels of resistance to both types (i.e., I and II) of pyrethroids. The prevalence of this mutation represents a new challenging factor for the control of A. aegypti.

\section{Results}

Characterization of the levels of resistance to type I and type II pyrethroids in larvae. The analyses of the concentration-mortality parameters were based on the low $\chi 2$-values $(<8.0)$ and high $P$-values $(>0.05)$ obtained using the probit model (Table 1). The lethal toxicities of each pyrethroid to the larvae of the susceptible strain were similar, with $\mathrm{LC}_{50}$ values that varied from 0.001 to $0.003 \mu \mathrm{g}$ of a.i. $/ \mathrm{mL}$ (Fig. 1). However, the resistance ratios (RR) to pyrethroids (compared to the $\mathrm{LC}_{50}$ for the larvae of the susceptible strain) was 59,830 $(38,150-93,830)$-fold to permethrin and 1,568 (772-3,186)-fold to deltamethrin (Table 1, Fig. 1A and B).

High levels of pyrethroid resistance in larvae are the result of both enhanced metabolic detoxification and $k d r$-mutations. The bioassays with the three synergists (PBO, TPP, and DEM) revealed that the high levels of resistance to both pyrethroids (i.e., permethrin and deltamethrin) were partially based on enzymatic detoxification (Fig. $1 \mathrm{C}$ and D). The mortality to permethrin at the $\mathrm{LC}_{50}$ of the resistant population was significantly increased only by $\mathrm{PBO}(d f=3 ; F=48.0 ; P<0.001)$ (Fig. $1 \mathrm{C})$, whilst all three synergists increased mortality to deltamethrin at the $\mathrm{LC}_{50}$ (Deltamethrin: $\left.d f=3 ; F=128.3 ; \mathrm{P}<0.001\right)$ (Fig. 1D).

High levels of pyrethroid resistance in adults are mainly due to $k d r$-mutations. For the results from the bioassays with adult mosquitoes, while the $\mathrm{LC}_{50}$ for permethrin in susceptible mosquitoes was $3.4 \times 10^{-3}\left(3.0 \times 10^{-3}-3.8 \times 10^{-3}\right) \mu$ g a.i. $/ \mathrm{cm}^{2}$ (Table 1, Fig. $\left.2 \mathrm{~A}\right)$, the $\mathrm{LC}_{50}$ for deltamethrin was $7.6 \times 10^{-6}$ $\left(6.6 \times 10^{-6}-8.8 \times 10^{-6}\right) \mu \mathrm{g}$ a.i. $/ \mathrm{cm}^{2}$ (Table 1, Fig. $2 \mathrm{~B}$ ). Based on these $\mathrm{LC}_{50}$ measures, permethrin was 243.2 (182.7-323.8)-fold less toxic than deltamethrin. Compared to the susceptible strain, the resistant strain was 102.2 (73.7-141.7)-fold more resistant to permethrin and 129.0 (88.4-188.3)-fold more resistant to deltamethrin (Table 1, Fig. 2A and B).

In contrast to results for the larval assays, pre-treatment with three synergists, PBO, TPP and DEM, did not enhance the toxicities of permethrin nor deltamethrin (Fig. 2C and D) in adult mosquitoes (Permethrin: $F=0.36$; $P=0.78$. Deltamethrin: $F=0.33 ; P=0.80$ ).

First record of $\mathrm{V} 410 \mathrm{~L}$ mutation (alone and together with F1534C mutation) in the sodium channel gene of $\boldsymbol{A}$. aegypti. To identify possible genetic modifications responsible for the high levels of pyrethroid resistance, the complete coding region of the sodium channel from the pyrethroid-resistant 


\section{concentration-mortatlity and synergism bioassays with larvae}

a

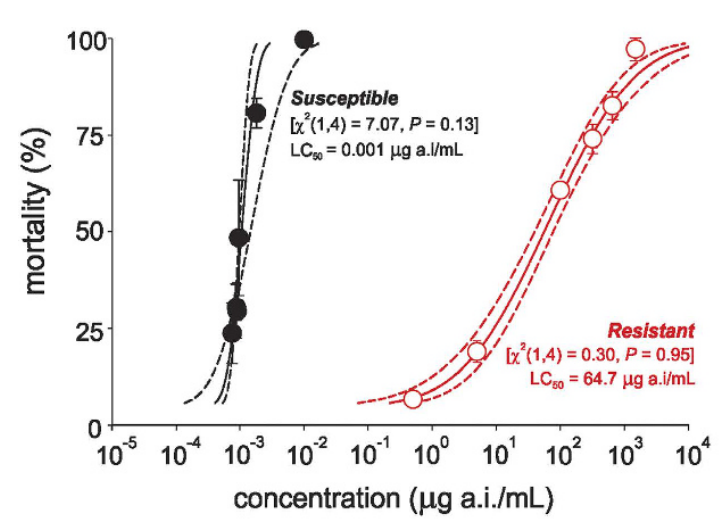

b

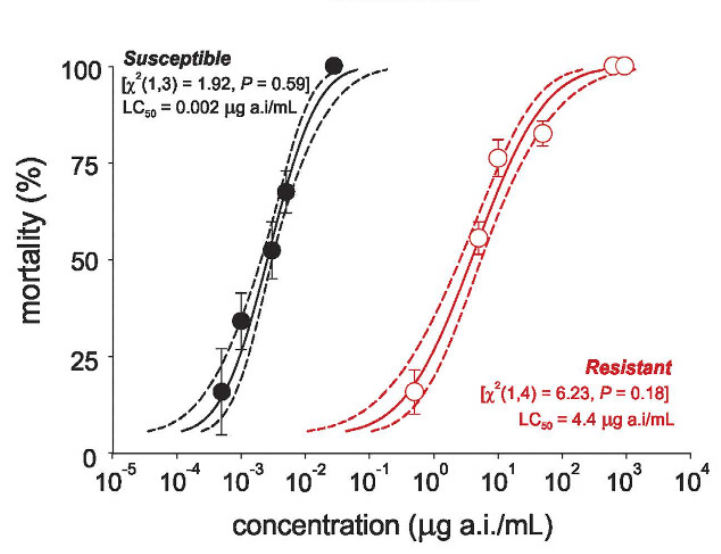

C

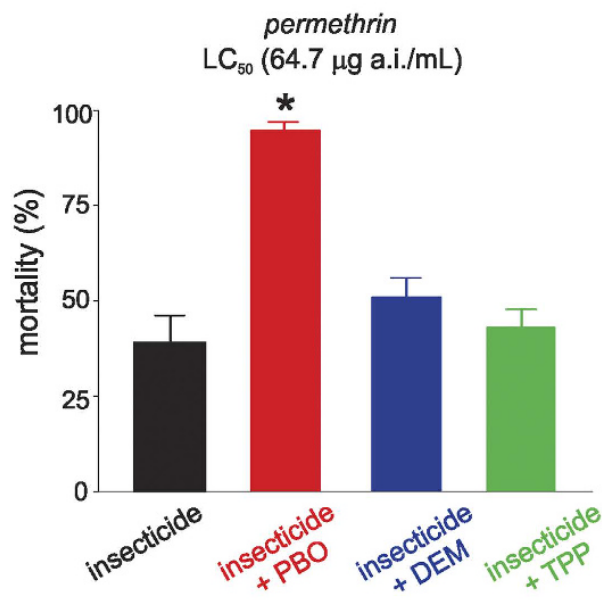

d

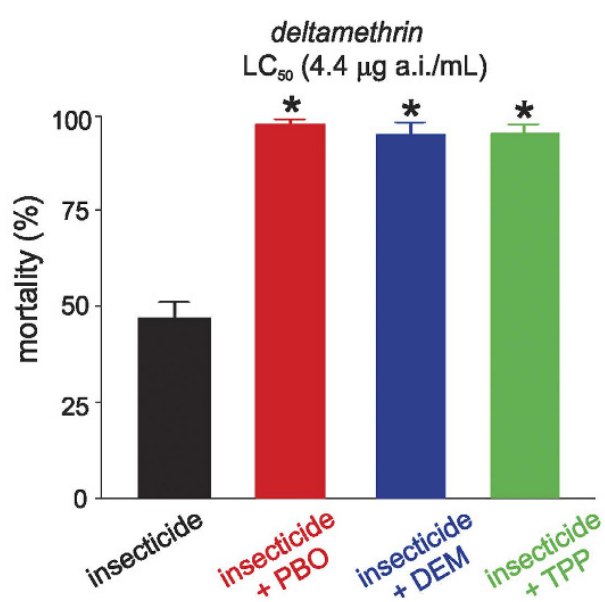

Figure 1. Toxicity (a,b) and comparative effects of synergists $(\mathbf{c}, \mathbf{d})$ on mortality caused by permethrin and deltamethrin to larvae (L4) of two Brazilian strains of Aedes aegypti. (a,b) The lines denote the lethal concentration (LC) values estimated based on concentration-mortality bioassays using probit analyses. Symbols show the averaged mortality for each insecticide concentration applied to each population of A. aegypti. The vertical bars represent the standard error of the average (SE). (c,d) The effects of the synergists piperonyl butoxide (PBO), diethyl maleate (DEM) and triphenyl phosphate (TPP) on the mortality of the resistant population caused by pyrethroids at the $\mathrm{LC}_{50}$ obtained for the resistant strain. Asterisks indicate significant differences in relation to the unsynergized insecticide (one-way ANOVA with Scheffe's post hoc analysis, $P<0.05)$.

(Accession number: KY747530) and pyrethroid-susceptible (Accession number: KY747529) strains was cloned and sequenced. Sequence comparison revealed eight nucleotide alterations between pyrethroid-resistant and pyrethroid-susceptible populations of which only two resulted in amino acid substitutions (Fig. 3A). One substitution was at position 410 in the transmembrane segment 6 of domain I (IS6) in which a valine was substituted by a leucine (V410L), and the other substitution was at position 1534 in the transmembrane segment 6 of domain III (IIIS6) in which a phenyl-alanine was substituted by a cysteine (F1534C). The comparison of genomic DNA sequences of 25 individual mosquitoes from both susceptible and resistant populations of A. aegypti confirmed these amino acid substitutions. Our results showed that all the sequenced pyrethroid resistant mosquitoes had the F1534C mutation and 7 of the 10 also carried the V410L mutation. The allele frequencies were 100 and $50 \%$ for both mutations, respectively.

V410L mutation confers AaNav1-1 channel resistance to both type I and type II pyrethroids. To assess the effects of the two mutations on pyrethroid resistance, we used the pyrethroid-sensitive mosquito sodium channel $\mathrm{AaNa}_{\mathrm{v}} 1-1^{20}$ to generate mutant channels expressing either single (i.e., V410L or F1534C) or double mutations (i.e., V410L + F1534C). Consistent with previous findings ${ }^{17,20,21}$, the F1534C mutation significantly decreased the AaNav1-1 sensitivity to permethrin (Fig. 3B) but not to deltamethrin (Fig. 3C). Interestingly, 
concentration-mortatlity and synergism bioassays with adults

a

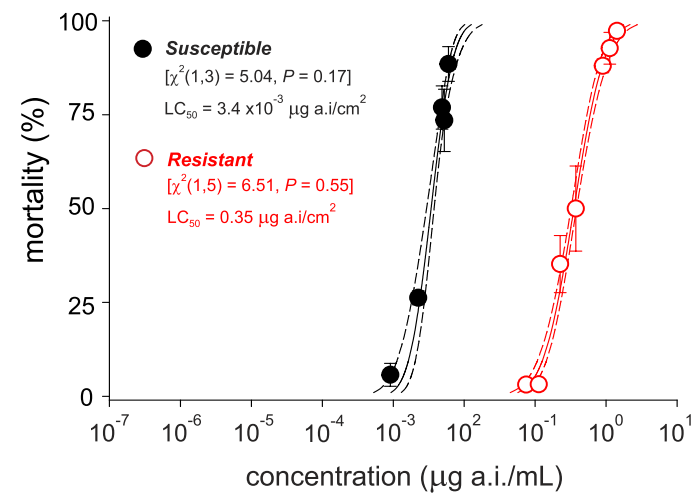

b

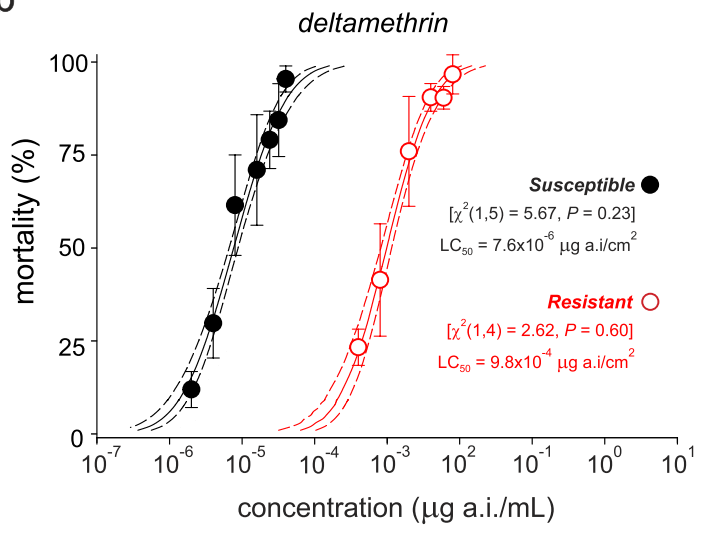

C permethrin $\mathrm{LC}_{50}\left(0.35 \mu \mathrm{g}\right.$ a.i. $\left./ \mathrm{cm}^{2}\right)$

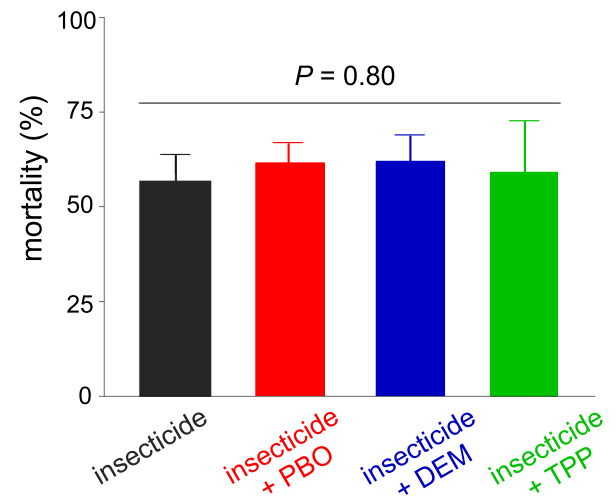

d

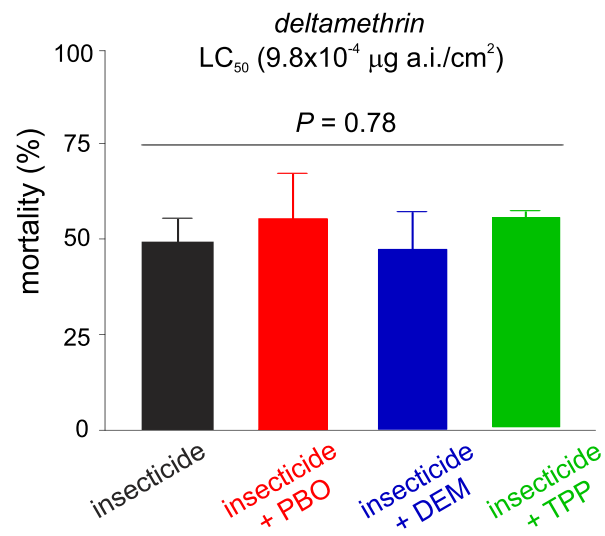

Figure 2. Toxicity (a,b) and comparative effects of synergists (c,d) on mortality caused by permethrin and deltamethrin to adults of two Brazilian strains of Aedes aegypti. (a,b) The lines denote the lethal concentration (LC) values estimated based on concentration-mortality bioassays using probit analyses. Symbols show the averaged mortality for each insecticide concentration applied to each population of $A$. aegypti. The vertical bars represent the standard error of the average (SE). (c,d) The effects of the synergists piperonyl butoxide (PBO), diethyl maleate (DEM) and triphenyl phosphate (TPP) on the mortality of the resistant population caused by pyrethroids at the $\mathrm{LC}_{50}$ obtained for the resistant strain. Asterisks indicate significant differences in relation to the unsynergized insecticide (one-way ANOVA with Scheffe's post hoc analysis, $P<0.05$ ).

the V410L mutation alone reduced the sensitivity of mosquito AaNav1-1 to both deltamethrin and permethrin by approximately 10-fold (Fig. 3B and C), and the V410L and F1534 double mutations further enhanced the resistance of $\mathrm{AaNa}_{\mathrm{v}} 1-1$ channels to permethrin compared with the two single mutations (Fig. 3B). These results indicate an additive effect of the two mutations on permethrin resistance.

Screening of wild populations for the presence of kdr mutations. The sequencing of two fragments harboring the $k d r$ mutations (V410L and F1534C) showed that none of the 11 field collected populations has the V410L mutation while the F1534C substitution was detected in five populations (Table 2). When present, the F1534C mutation was always heterozygous (Table 2).

\section{Discussion}

In this study, we report on a Brazilian strain of $A$. aegypti with high levels of pyrethroid resistance conferred primarily by $k d r$ (i.e., V410L and F1534C) mutations, especially in adult mosquitoes. The V410L mutation is reported for the first time in the sodium channels of mosquitoes, and by identifying the valine to leucine substitution at a corresponding position in a mosquito (i.e., $\mathrm{AaNa}_{\mathrm{v}} 1-1$ ) sodium channel, we demonstrated that this amino acid substitution reduced the sensitivity of these channels to both type I (i.e., permethrin) and type II pyrethroids (i.e., deltamethrin).

Vector control has long been a key strategy in managing mosquito-borne human diseases, and the application of pyrethroid insecticides continues to be one of most important components in this effort ${ }^{1-5}$. However, 
a

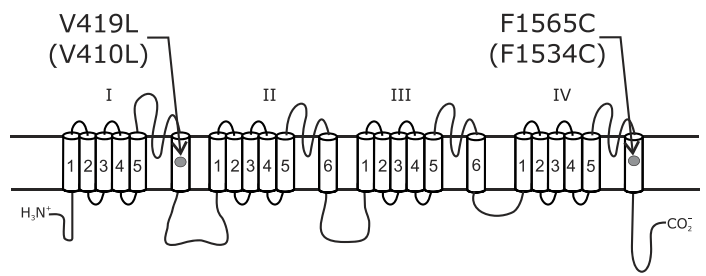

b

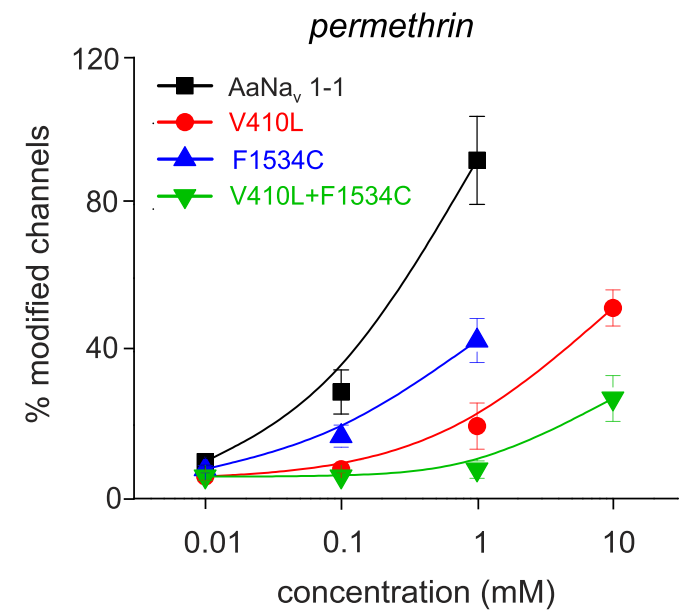

C

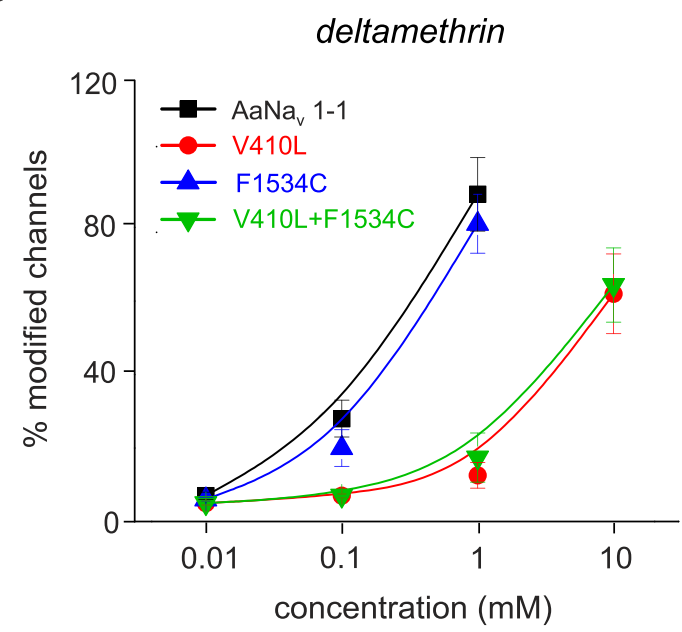

Figure 3. Pyrethroid resistance-associated amino acid substitutions in A. aegypti sodium channels. (a) Schematic representation of the mosquito sodium channel protein $\left(\mathrm{AaNa}_{\mathrm{v}} 1-1\right)$ indicating two knockdown resistance mutations in IS6 and IVS6. Sodium channels are large transmembrane proteins with four homologous repeats (I-IV), each having six transmembrane segments (S1-S6). Residue positions correspond to the cockroach $\mathrm{BgNa}_{\mathrm{v}}$ protein (GenBank number: U73583). Dose-response curves of permethrin (b) and deltamethrin (c) on BgNav1-1a, V410L, F1534C and V410L + F1534C cockroach sodium channels. The method for determining the percentage of modified channels is described in the Materials \& Methods section.

the development of pyrethroid resistance is a major threat to the sustained use of pyrethroids in vector control. Historically, resistance has developed in Brazilian populations of disease-vector mosquitoes to a wide range of insecticides, including pyrethroids ${ }^{16,22-25}$.

Although more than 50 sodium channel mutations are associated with pyrethroid resistance in diverse arthropod pests, not all of the mutations cause resistance by reducing the docking of the pyrethroid at the sodium channels ${ }^{7,11,19,20}$. Such was likely the case for the V410L mutation because the amino acid substitution was not located in one of the two recently described pyrethroid receptor sites ${ }^{11,19,20,26}$. One potential alternative explanation is that some $k d r$ mutations reduce pyrethroid effects by altering the gating properties of the channel without inhibiting molecule docking. This altered gating could contribute to resistance because pyrethroids prefer binding to sodium channels in an open state, and $k d r$ mutations that deter gating transitions to the open state would counteract the 


\begin{tabular}{|l|c|c|}
\hline \multirow{2}{*}{ Strains } & \multicolumn{2}{|c|}{$\boldsymbol{k d}$ mutations } \\
\cline { 2 - 3 } Cedro & V410L & F1534C \\
\hline Recife & GTA & TGC \\
\hline Santa Cruz do Capeberibe & GTA & TGC \\
\hline São José do Egito & GTA & TGC \\
\hline Afogados da Ingazeira & GTA & TGC \\
\hline Serra Talhada & GTA & TTC \\
\hline Itaíba & GTA & TTC \\
\hline Arcoverde & GTA & TGC \\
\hline Gloria do Goitá & GTA & TTC \\
\hline
\end{tabular}

Table 2. Frequencies of two $k d r$ mutations (V410L and F1534C) in 11 Brazilian populations of Aedes aegypti sampled from Pernambuco state and reported resistant to type II pyrethroid. (V: GTA; L: TTA; F: TTC; C: TGC).

pyrethroid effects ${ }^{9}$. For example, altered kinetics of channel gating and reduced channel sensitivity to pyrethroids are reported when an aspartic acid (D) is replaced by a lysine $(\mathrm{K})$ at position 802 in IIS1 in a cockroach (BgNav) splicing variant sodium channel, in addition to when valine to leucine or isoleucine substitutions occur at the 410 corresponding position in a cockroach sodium channel (BgNav1-1a) ${ }^{27,28}$.

More than one $k d r$ mutation associated with insecticide resistance are frequently found in insects ${ }^{7,13}$, including in A. aegyptiti,16-18. The occurrence of both V410L and F1534C mutations might explain the very high levels of resistance found in this study, indicating that monitoring strategies should focus on the screening of more than one sodium channel region. For example, the allele frequencies for the F1534C mutation were consistent with the wide distribution of F1534C previously reported in Brazil ${ }^{25}$, which would support the mosquito control programs that integrate the rotations between type I and type II pyrethroid products as a control strategy. This strategy could be successful because the F1534C mutation is confirmed to reduce the sensitivity of A. aegypti AaNav1-1 channels to type I (e.g., permethrin) but not to type II (e.g., deltamethrin) pyrethroids ${ }^{17,21}$. However, the very high levels of pyrethroid insensitivity conferred by the V410L mutation alone or in combination with F1534C could make pyrethroids not only largely ineffective in Brazilian regions in which A. aegypti have these mutations in combination but could also make the situation worse by increasing the pressure to select resistant individuals.

The screening of field populations from the Brazilian Pernambuco state revealed the absence of the mutation V410L which may indicate that this mutation is not widely spread in natural populations. Furthermore, half of the same samples were found to harbor the widely spread F1534C substitution, which helps to explain the resistance mechanisms for these mosquitoes populations ${ }^{29}$. The pyrethroids resistance in these populations was reported to be correlated not only with different $k d r$ mutations (e.g., the $\mathrm{I} 1011 \mathrm{M}$ ) but also with detoxification mechanisms ${ }^{29}$, indicating clearly the contribution of various mechanisms to the reported cases of pyrethroid resistance in Brazil.

In the present investigation, for instance, we demonstrated the contribution of various mechanisms to the high level of pyrethroid resistance in the resistant strain, especially in larvae. The pre-exposure of larvae from the resistant strain to the synergist $\mathrm{PBO}$ increased the mortality caused by both deltamethrin or permethrin alone, which indicate the involvement of the cytochrome P450-dependent monooxygenases ${ }^{30-33}$ and other targets (e.g., esterases) secondarily affected by this synergist ${ }^{32,34}$. Furthermore, based on our results from the synergism bioassays with TPP and DEM and as previously demonstrated in insects ${ }^{35}$, the esterases and also glutathione-S-transferases do also contribute to the high resistance level to deltamethrin in larvae of the resistant strain. However, the high resistance level recorded for adults of the resistant strain is mainly due to $k d r$-mutations as the pre-treatment of the three synergists did not increase the toxicities of permethrin and deltamethrin in adult mosquitoes. It is clear that there are enormous gaps in our knowledge of insect enzymes.

Thus, further investigations aiming to identify the distribution of the V410L mutation in Brazilian populations of A. aegypti and on the correlation of the mutation with pyrethroid resistance in field populations are warranted. Additionally, the effects of this mutation on the ability of mosquitoes to transmit human arboviruses also require investigation.

\section{Materials and methods}

This study did not use any live vertebrates and followed the laws and ethical guidelines of Brazil. Furthermore, owing to the nature of specimens used (i.e. this study did not involve any endangered or protected species) and as all mosquitoes used were reared at the insectary of the Department of General Biology, Federal University of Viçosa (Viçosa, MG, Brazil), no additional permissions or approvals were required.

Mosquito strains used in the concentration mortality bioassays. The two strains used in the concentration-mortality bioassays were the pyrethroid-susceptible PPCampos population (Campos dos Goytacazes, Rio de Janeiro State, Brazil) and the pyrethroid-resistant F2 Oiapoque population (Rio de Janeiro, Rio de Janeiro State, Brazil). Mosquito strains were maintained in the insectary of the Department of General Biology, Federal University of Viçosa (Viçosa, MG, Brazil), under controlled conditions of temperature $\left(25 \pm 3^{\circ} \mathrm{C}\right)$, relative humidity $(60 \pm 2 \%)$, and photoperiod (12:12 L:D), as previously described in Marriel et al. ${ }^{36}$. 
Characterization of mosquito sensitivity to pyrethroids. We conducted concentration mortality bioassays using larvae and adults. The concentration-mortality bioassays using mosquito were conducted based on the protocol recommended by $\mathrm{WHO}^{37}$. In brief, five repetitions of 25 early fourth instar larvae (L4) were put in glass jars $(200 \mathrm{~mL})$ with $100 \mathrm{~mL}$ of insecticide solutions at various concentrations, which caused 5-95\% mortality. The control group was maintained in $100 \mathrm{ml}$ of distilled water. Mortality was recorded $24 \mathrm{~h}$ after treatment. At least 6 different concentrations were used for each insecticide. We used commercial formulations of the pyrethroids permethrin (25 g a.i./L, BASF Brasil, São Paulo - SP, Brazil) and deltamethrin ( $25 \mathrm{~g}$ a.i./L, Bayer Crop Science, São Paulo - SP, Brazil).

For the concentration-mortality bioassays with adults, glass-vial based bioassays were carried out. The inner wall of $200 \mathrm{~mL}$ glass vials was impregnated with $2 \mathrm{~mL}$ of insecticide solution at various concentrations and left to dry by rotation for $2 \mathrm{~h}$. At least five different concentrations were used for each insecticide. 25 to 30 one- to two-day old starved females were exposed in each vial and four vials were used for each insecticide concentration. Mortality was assessed after $24 \mathrm{~h}$ exposure.

Bioassays using synergists that inhibit detoxification enzymes. The contribution of enzymatic detoxification was quantified by measuring the mortality of resistant L4 as well as adults that were previously exposed to the following synergists: triphenyl phosphate (TPP; an esterase inhibitor), diethyl maleate (DEM; a glutathione S-transferase inhibitor), and piperonyl butoxide (PBO; an inhibitor of cytochrome P450-dependent monooxygenases and esterases). Preliminary tests with varying concentrations of synergists were carried out to determine the maximum sublethal concentrations for adult and larvae mosquitoes. Subsequently, these maximum sublethal concentrations were used in the synergism tests were of $1.5 \%, 2.5$ and $2 \%$ for PBO, DEM, and TPP, respectively.

For the bioassays using larvae, the synergists were dissolved in acetone, and $1 \mathrm{~mL}$ of the synergist solutions was added to $99 \mathrm{~mL}$ of distilled water. Twenty-five early L4 were transferred to glass jars and remained in contact with a synergist for $1 \mathrm{~h}$ before being exposed to an insecticide solution (at the corresponding lethal concentration capable of killing $50 \%$ of tested larvae - $\mathrm{LC}_{50}$ ). The $\mathrm{L} 4$ exposed to synergists were then transferred to other glass jars containing $100 \mathrm{~mL}$ of insecticide solutions. Two control groups were used, one with only $100 \mathrm{~mL}$ of distilled water and the other with the addition of $1 \mathrm{ml}$ acetone into to $99 \mathrm{~mL}$ of distilled water. Mortality was recorded $24 \mathrm{~h}$ after insecticide treatment.

For adults, the bioassay was similar to the one for the concentration mortality. The inner wall of $200 \mathrm{~mL}$ glass vials was impregnated, at various concentrations, with $2 \mathrm{~mL}$ of insecticide solution containing acetone dissolved synergists. As controls, we used vials impregnated with water alone, with water and acetone. Mortality was recorded $24 \mathrm{~h}$ after insecticide treatment.

Cloning and sequencing of CDNA and genomic DNA of the sodium channel gene from $A$. aegypti. Total RNA from pools of 15-20 adult mosquitoes of each population was extracted using a PureLink ${ }^{\circledR}$ RNA Mini Kit according to the manufacturer's protocol (Life Technologies, Carlsbad, California, USA). Subsequently, first-strand cDNA was synthesized from total RNA using SuperScript III reverse transcriptase (Invitrogen, Carlsbad, California, USA). PCRs were performed with specific primer pairs designed based on the A. aegypti sequence available in GenBank (accession number AY663385) (Supplementary Table S1). PCR reactions $(20 \mu \mathrm{L})$ consisted of $1.2 \mu \mathrm{L}$ of template cDNA, $0.75 \mu \mathrm{L}$ of each primer $(10 \mu \mathrm{M}), 12 \mu \mathrm{l}$ of GreenTaq (Fermentas, Waltham, Massachusetts, USA) and $6 \mu \mathrm{l}$ of sterile distilled water. PCR cycling conditions were as follow: $94^{\circ} \mathrm{C}$ for $2 \mathrm{~min} ; 35 \mathrm{cycles}$ at $94^{\circ} \mathrm{C}$ for $45 \mathrm{~s}$, at $58^{\circ} \mathrm{C}$ for $60 \mathrm{~s}$, and at $72^{\circ} \mathrm{C}$ for $120 \mathrm{~s}$; and $72{ }^{\circ} \mathrm{C}$ for $7 \mathrm{~min}$. The amplified fragments of the expected size were purified using Wizard SV gel and PCR Cleanup System from Promega (Madison, Wisconsin, USA) and then cloned using Stellar ${ }^{\mathrm{TM}}$ Competent Cells from Clonetech (Mountain View, California, USA) according to the manufacturer's instructions. Five to six DNA clones for each segment were sent for sequencing at Macrogen facilities (Macrogen Inc., Seoul, South Korea).

To confirm the amino acid substitution mutations, genomic DNA was extracted separately from 10 insects. Primers flanking the potential mutation sites (Supplementary Table S1) were designed. PCRs were performed as follow: $94^{\circ} \mathrm{C}$ for $2 \mathrm{~min} ; 35$ cycles at $94^{\circ} \mathrm{C}$ for $40 \mathrm{~s}$, at $58^{\circ} \mathrm{C}$ for $45 \mathrm{~s}$, and at $72^{\circ} \mathrm{C}$ for $75 \mathrm{~s}$; and $72^{\circ} \mathrm{C}$ for $10 \mathrm{~min}$. PCR products were directly sequenced (using internal primers) as described above.

Functional characterization of the sensitivity of the AaNav1-1 and mutant mosquito sodium channels to pyrethroids in Xenopus oocytes. To introduce V410L, F1534C and V410L + F1534C mutations into a pyrethroid-sensitive mosquito sodium channel (AaNav1-1), site-directed mutagenesis was performed with Polymerase Chain Reaction (PCR) using Pfu Turbo DNA polymerase (Stratagene, La Jolla, CA, USA). All mutagenesis results were confirmed by DNA sequencing. Procedures for oocytes preparation and cRNA injection were identical to those described previously ${ }^{38}$. For robust expression of AaNav1-1 sodium channels, cRNA was co-injected into oocytes with cRNA encoding the Drosophila melanogaster tipE auxiliary subunit (1:1 ratio), which enhances the expression of insect sodium channels in oocytes ${ }^{39,40}$.

To quantify the effects of type I (i.e., permethrin) and type II (i.e., deltamethrin) pyrethroids, a two-electrode voltage clamp was used to measure pyrethroid-induced tail currents. Methods and data analysis for the two-electrode voltage clamp recording of sodium currents and measurement of tail currents induced by pyrethroids were identical to those previously described ${ }^{38,41}$. Briefly, the pyrethroid-induced tail current was recorded during a 100-pulse train of 5-ms step depolarizations from -120 to $0 \mathrm{mV}$ with 5-ms interpulse intervals $^{42}$. The percentage of channels modified by pyrethroids was calculated as follows: $M=\left\{\left[\mathrm{I}_{\text {tail }} /\left(\mathrm{E}_{\mathrm{h}}-\mathrm{E}_{\mathrm{Na}}\right)\right] /\left[\mathrm{I}_{\mathrm{Na}} /\right.\right.$ $\left.\left.\left(\mathrm{E}_{\mathrm{t}}-\mathrm{E}_{\mathrm{Na}}\right)\right]\right\} \times 100^{41}$, where $\mathrm{I}_{\text {tail }}$ is the maximal tail current amplitude, $\mathrm{E}_{\mathrm{h}}$ is the potential to which the membrane is repolarized, $\mathrm{E}_{\mathrm{Na}}$ is the reversal potential for sodium current determined from the current-voltage curve, $\mathrm{I}_{\mathrm{Na}}$ 
is the amplitude of the peak current during depolarization before pyrethroid exposure, and $\mathrm{E}_{\mathrm{t}}$ is the potential of step depolarization.

All experiments were performed at room temperature. Sodium currents were measured with an OC725C oocyte clamp (Warner Instruments, Hamden, CT) and a Digidata $1440 \mathrm{~A}$ interface (Axon Instruments Inc., Foster City, CA), and pCLAMP 10.2 software (Axon Instruments Inc.) was used for data acquisition and analysis. Deltamethrin and permethrin were kindly provided by Dr. Bhupinder Khambay (Rothamsted Research Institute, Hertfordshire, UK) and Dr. Ralf Nauen (Bayer CropScience AG, Monheim, Germany). The purities of different pyrethroids ranged from $99.3 \%$ to $99.8 \%$. Stock solutions of pyrethroids $(100 \mathrm{mM})$ were dissolved in dimethyl sulfoxide (DMSO). The working concentration was prepared in ND96 recording solution immediately before the experiments. The concentration of DMSO in the final solution was $<0.5 \%$, which had no effect on the function of sodium channels in the experiments.

Screening of wild populations for the presence of kdr mutations. DNA samples of eleven field collected populations from Pernambuco state in the Brazilian Northeast region were generously obtained from the Departamento de Entomologia/CPqAM/Fiocruz-PE (Supplementary Table S2). The populations were initially established from eggs collected in "oviposition traps" in various municipalities of Pernambuco state. Their susceptibility to the pyrethroid type II cypermethrin were previously assessed and the resistance levels ranged from 7.1 to 222.6 -fold ${ }^{29}$. We used the specific primers flanking the two mutation sites (Supplementary Table S1) under the same described PCRs conditions and PCR products were directly sequenced (using internal primers) as described above.

Statistical Analyses. Concentration-mortality data were subjected to probit analysis (PROC PROBIT; SAS Institute 2008), and 95\% CIs for resistance ratios (RRs) were estimated as reported by Robertson et al. ${ }^{43}$ and considered significant when they did not include the value 1 . The synergism results and those for the sodium channel sensitivity were subjected to one-way analysis of variance (one-way ANOVA) and to Scheffe's post hoc analysis test $(P<0.05)$ to isolate the different treatments, using SigmaPlot 12.0 (Systat Software, San Jose, CA, USA).

Ethical approval. All applicable international, national, and institutional guidelines for the care and use of animals were considered in the present investigation.

\section{References}

1. Liu, N. Insecticide resistance in mosquitoes: Impact, mechanisms, and research directions. Annual Review of Entomology 60, 537-559, doi: 10.1146/annurev-ento-010814-020828 (2015).

2. Reiter, P. Control of urban Zika vectors: Should we return to the successful PAHO/WHO strategy? Plos Negl. Trop. Dis 10, e0004769 (2016).

3. Devine, G. J. et al. Using adult mosquitoes to transfer insecticides to Aedes aegypti larval habitats. Proceedings of the National Academy of Sciences 106, 11530-11534, doi: 10.1073/pnas.0901369106 (2009).

4. WHO. Vector control operations framework for Zika virus. Geneva: World Health Organization (2016).

5. WHO. An operational manual for indoor residual spraying (IRS) for malaria transmission control and elimination. Geneva: World Health Organization (2013).

6. Soderlund, D. M. State-dependent modification of voltage-gated sodium channels by pyrethroids. Pesticide biochemistry and physiology 97, 78-86 (2010).

7. Dong, K. et al. Molecular biology of insect sodium channels and pyrethroid resistance. Insect biochemistry and molecular biology 50, 1-17 (2014).

8. Soderlund, D. M. Molecular mechanisms of pyrethroid insecticide neurotoxicity: recent advances. Archives of toxicology 86, 165-181 (2012)

9. Silver, K. S. et al. Voltage-gated sodium channels as insecticide targets. Advances in insect physiology 46, 389-433 (2014).

10. Smith, L. B., Kasai, S. \& Scott, J. G. Pyrethroid resistance in Aedes aegypti and Aedes albopictus: Important mosquito vectors of human diseases. Pesticide Biochemistry and Physiology (2016)

11. Zhorov, B. S. \& Dong, K. Elucidation of pyrethroid and DDT receptor sites in the voltage-gated sodium channel. NeuroToxicology, doi: http://dx.doi.org/10.1016/j.neuro.2016.08.013 (2016).

12. Ranson, H. \& Lissenden, N. Insecticide resistance in African Anopheles Mosquitoes: A worsening situation that needs urgent action to maintain malaria control. Trends in Parasitology 32, 187-196, doi: http://dx.doi.org/10.1016/j.pt.2015.11.010 (2016).

13. Rinkevich, F. D., Du, Y. \& Dong, K. Diversity and convergence of sodium channel mutations involved in resistance to pyrethroids. Pesticide biochemistry and physiology 106, 93-100 (2013).

14. Soderlund, D. M. In Comprehensive molecular insect science: Pharmacology Vol. 5, (eds Gilbert, L. I., Iatrou, K. \& Gill, S. S.) 1-24 (Elsevier, 2005)

15. Davies, T., Field, L., Usherwood, P. \& Williamson, M. A comparative study of voltage-gated sodium channels in the Insecta: implications for pyrethroid resistance in Anopheline and other Neopteran species. Insect molecular biology 16, 361-375 (2007).

16. Brito, L. P. et al. Assessing the effects of Aedes aegypti kdr mutations on pyrethroid resistance and its fitness cost. PloS One 8, e60878 (2013).

17. Hirata, K. et al. A single crossing-over event in voltage-sensitive $\mathrm{Na}^{+}$channel genes may cause critical failure of dengue mosquito control by insecticides. PLoS Negl Trop Dis 8, doi: 10.1371/journal.pntd.0003085 (2014).

18. Jones, C. M. et al. Footprints of positive selection associated with a mutation (N1575Y) in the voltage-gated sodium channel of Anopheles gambiae. Proceedings of the National Academy of Sciences 109, 6614-6619 (2012).

19. Du, Y., Nomura, Y., Zhorov, B. S. \& Dong, K. Rotational symmetry of two pyrethroid receptor sites in the mosquito sodium channel. Molecular pharmacology 88, 273-280 (2015).

20. Du, Y. et al. Molecular evidence for dual pyrethroid-receptor sites on a mosquito sodium channel. Proc Natl Acad Sci USA 110, doi: $10.1073 /$ pnas.1305118110 (2013).

21. Hu, Z., Du, Y., Nomura, Y. \& Dong, K. A sodium channel mutation identified in Aedes aegypti selectively reduces cockroach sodium channel sensitivity to type I, but not type II pyrethroids. Insect biochemistry and molecular biology 41, 9-13 (2011).

22. Belinato, D. F. et al. Resistance Status to the Insecticides Temephos, Deltamethrin, and Diflubenzuron in Brazilian Aedes aegypti Populations. BioMed Research International 2016 (2016).

23. Chapadense, F. G. G. et al. Phenotypic and genotypic profile of pyrethroid resistance in populations of the mosquito Aedes aegypti from Goiania, Central West Brazil. Revista da Sociedade Brasileira de Medicina Tropical 48, 607-609 (2015). 
24. Chediak, M. et al. Spatial and temporal country-wide survey of temephos resistance in Brazilian populations of Aedes aegypti. Memórias do Instituto Oswaldo Cruz 111, 311-321 (2016).

25. Linss, J. G. et al. Distribution and dissemination of the Val1016Ile and Phe1534Cys Kdr mutations in Aedes aegypti Brazilian natural populations. Parasit Vectors 7, doi: 10.1186/1756-3305-7-25 (2014).

26. O'Reilly, A. O. et al. Modelling insecticide-binding sites in the voltage-gated sodium channel. Biochemical Journal 396, 255-263 (2006).

27. Du, Y. et al. A negative charge in transmembrane segment 1 of domain II of the cockroach sodium channel is critical for channel gating and action of pyrethroid insecticides. Toxicology and Applied Pharmacology 247, 53-59, doi: http://dx.doi.org/10.1016/j. taap.2010.05.016 (2010).

28. Oliveira, E. E., Du, Y., Nomura, Y. \& Dong, K. A residue in the transmembrane segment 6 of domain I in insect and mammalian sodium channels regulate differential sensitivities to pyrethroid insecticides. Neurotoxicology 38, 42-50 (2013).

29. Araújo, A. P. Análise da resistência a inseticidas químicos em populações de Aedes aegypti (Diptera: Culicidae), de Municípios do Estado de Pernambuco PhD thesis, Fundação Oswaldo Cruz, Recife-PE, 120p (2013).

30. Scott, J. G. Cytochromes P450 and insecticide resistance. Insect Biochemistry and Molecular Biology 29, 757-777, doi: http://dx.doi. org/10.1016/S0965-1748(99)00038-7 (1999)

31. Feyereisen, R. In Comprehensive Molecular Insect Science vol. 4, Elsevier, Oxford, pp. 1-77. (eds Gilbert, L. I., Latrou, K. \& Gill, S.) (2005).

32. Hemingway, J., Hawkes, N. J., McCarroll, L. \& Ranson, H. The molecular basis of insecticide resistance in mosquitoes. Insect Biochemistry and Molecular Biology 34, 653-665, doi: http://dx.doi.org/10.1016/j.ibmb.2004.03.018 (2004).

33. Liu, N., Li, T., Reid, W., Yang, T. \& Zhang, L. Multiple cytochrome P450 Genes: Their constitutive overexpression and permethrin induction in insecticide resistant mosquitoes, Culex quinquefasciatus. PLoS ONE 6, e23403 (2011).

34. Ranson, H. \& Hemingway, J. In Comprehensive Molecular Insect Science, vol. 5, Elsevier, Oxford, pp. 383-402. (eds Gilbert, L. I., Latrou, K. \& Gill, S.) (2005).

35. Hemingway, J., Field, L. \& Vontas, J. An overview of insecticide resistance. Science 298, 96-97, doi: 10.1126/science.1078052 (2002).

36. Marriel, N. B., Tomé, H. V. V., Guedes, R. C. N. \& Martins, G. F. Deltamethrin-mediated survival, behavior, and oenocyte morphology of insecticide-susceptible and resistant yellow fever mosquitos (Aedes aegypti). Acta Tropica 158, 88-96, doi: http:// dx.doi.org/10.1016/j.actatropica.2016.02.021 (2016).

37. WHO. Guidelines for laboratory and field testing of mosquito larvicides. Geneva: World Health Organization (2005).

38. Tan, J. et al. Identification of amino acid residues in the insect sodium channel critical for pyrethroid binding. Molecular pharmacology 67, 513-522 (2005).

39. Feng, G., Deak, P., Chopra, M. \& Hall, L. M. Cloning and functional analysis of TipE, a novel membrane protein that enhances Drosophila para sodium channel function. Cell 82, 1001-1011 (1995).

40. Warmke, J. W. et al. Functional expression of Drosophila para sodium channels modulation by the membrane protein TipE and toxin pharmacology. The Journal of general physiology 110, 119-133 (1997).

41. Tatebayashi, H. \& Narahashi, T. Differential mechanism of action of the pyrethroid tetramethrin on tetrodotoxin-sensitive and tetrodotoxin-resistant sodium channels. Journal of Pharmacology and Experimental Therapeutics 270, 595-603 (1994).

42. Vais, H. et al. Activation of Drosophila sodium channels promotes modification by deltamethrin reductions in affinity caused by knock-down resistance mutations. The Journal of general physiology 115, 305-318 (2000).

43. Robertson, J. L., Russel, R. M., Preisler, H. K. \& Savin, N. E. Pesticide Bioassays with Arthopods (2007).

\section{Acknowledgements}

We thank Dr. Ademir de Jesus Martins Júnior and José Bento Pereira Lima (Instituto Oswaldo Cruz, IOCFiocruz, Rio de Janeiro) for kindly providing the mosquito eggs of the F2 Oiapoque population. We thank also Dr. Constância Flávia Junqueira Ayres Lopes (CPqAM/Fiocruz-PE) for generously giving the DNA samples, Grants from the Science without Borders Program of the CAPES Foundation and the National Council of Scientific and Technological Development (CNPq) to KH; grants from the Arthur Bernardes Foundation (FUNARBE), the Minas Gerais State Foundation for Research Aid (FAPEMIG) and the National Council of Scientific and Technological Development (CNPq) to EEO, GFM, HVVT and WRV; and grants from the National Institute of Health (NIH) to KD and YD supported this work.

\section{Author Contributions}

E.E.O., K.H., H.V.V.T. and K.D. conceived/designed the research. K.H., H.V.V.T., Y.D., W.R.V., and Y.N. conducted the experiments. K.D., G.F.M. and E.E.O. contributed new reagents and/or analytical tools. E.E.O., K.H., H.V.V.T., W.R.V., and Y.D. analyzed the data. E.E.O., G.F.M., K.H. and K.D. wrote the manuscript. All authors read, corrected and approved the manuscript.

\section{Additional Information}

Supplementary information accompanies this paper at http://www.nature.com/srep

Competing Interests: The authors declare no competing financial interests.

How to cite this article: Haddi, K. et al. Detection of a new pyrethroid resistance mutation (V410L) in the sodium channel of Aedes aegypti: a potential challenge for mosquito control. Sci. Rep. 7, 46549; doi: 10.1038/ srep46549 (2017).

Publisher's note: Springer Nature remains neutral with regard to jurisdictional claims in published maps and institutional affiliations.

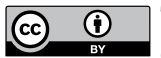

This work is licensed under a Creative Commons Attribution 4.0 International License. The images or other third party material in this article are included in the article's Creative Commons license, unless indicated otherwise in the credit line; if the material is not included under the Creative Commons license, users will need to obtain permission from the license holder to reproduce the material. To view a copy of this license, visit http://creativecommons.org/licenses/by/4.0/

(C) The Author(s) 2017 